\title{
All-optical switching in an optofluidic polydimethylsiloxane: Liquid crystal grating defined by cast-molding
}

\author{
Luciano De Sio, ${ }^{1,2, a)}$ Julien G. Cuennet, ${ }^{1}$ Andreas E. Vasdekis, ${ }^{1, b)}$ and Demetri Psaltis ${ }^{1}$ \\ ${ }^{1}$ Optics Laboratory, School of Engineering, Swiss Federal Institute of Technology Lausanne (EPFL), \\ CH-1015 Lausanne, Switzerland \\ ${ }^{2}$ LICRYL (Liquid Crystals Laboratory), National Institute for the Physics of Matter (INFM-CNR), \\ Center of Excellence CEMIF.CAL and Department of Physics, University of Calabria, \\ 87036 Arcavacata di Rende (CS), Italy
}

(Received 20 January 2010; accepted 9 March 2010; published online 1 April 2010)

\begin{abstract}
We report an optofluidic photoswitchable grating, based on a polydimethylsiloxane periodic structure on a glass substrate, separated by a thin liquid crystal film. The polydimethylsiloxane microstructure was realized via high resolution replica molding and was employed to both confine and align a photosensitive nematic liquid crystal. In the absence of any surface treatment, the liquid crystal exhibited homeotropic alignment. By inducing planar alignment on the glass substrate, a hybrid orientation of the liquid crystal was achieved, inducing polarization sensitive transmission. The photosensitivity of the liquid crystal enabled the all-optical control of the grating transmission and 20\% diffraction efficiency was measured. (C) 2010 American Institute of Physics.
\end{abstract}

[doi:10.1063/1.3377801]

Light responsive materials are promising candidates for the realization of optically addressed devices. In particular, dye-doped nematic liquid crystal (NLC) has attracted significant attention for its potential applications in the areas of all-optical holographic gratings ${ }^{1,2}$ and optical phase conjugation. ${ }^{3}$ These light sensitive liquid crystals (LC) contain photochromic molecules, which can undergo reversible trans-cis isomerization. This transition can be induced with $\mathrm{UV} / \mathrm{visible}$ light and reversed by heating or irradiation with light at longer wavelength. ${ }^{4,5}$ The combination of the NLC properties with on-chip photonic structures have been widely explored, ${ }^{6}$ however the integration of NLCs with costeffective and high-resolution nanostructures remains an open challenge. To this end, polydimethylsiloxane (PDMS) has attracted significant attention as a high-quality optical material, namely, due to its transparency, low surface energy, low dielectric constant and Young's modulus, and thermally and optically enabled polymerization. The emergence of optofluidics, where microfluidics are integrated with optics, has sparked renewed interest in PDMS for optical applications. ${ }^{7,8}$

The birefringent properties of LCs with the enhanced optical characteristics of PDMS have been recently combined to realize tuneable high-Q microresonators and transmission gratings. ${ }^{9,10}$ In these, the NLC is dispersed in a PDMS matrix and the photonic structure was defined either via self-assembly, or holographically, placing thus a limit in the morphology and shape control. In this letter, we report the integration of NLCs with a PDMS microstructure realized by cast-molding lithography and demonstrate an optically controlled transmission grating. Such hybrid device combines the advantage of cost-effective and high resolution diffractive structures with the tuneability of LCs. Its applications may involve optical switching or lab-on-a-chip optofluidic applications. The construction strategy of the device was based on high-resolution cast molding lithography,

\footnotetext{
${ }^{a)}$ Electronic mail: luciano.desio@ fis.unical.it. Tel.: 00390984496152.

${ }^{b)}$ Electronic mail: andreas.vasdekis@epfl.ch. Tel.: 0041216933742.
}

opening thus an alternative path toward cost-effective and highly multiplexed optical functionalities for lab-on-a-chip and information processing applications. ${ }^{11}$ We found that the low surface energy of the PDMS in combination with a "rubbed" bottom surface enabled a hybrid orientation of the $\mathrm{LC}$ and thus a polarization dependent transmission. We employed this finding to all-optically control the transmission of the hybrid grating.

As shown in Fig. 1(a), the device consisted of a PDMS grating and a modified glass substrate, with the NLC "sandwiched" between the two. The NLC was the E7 (Merck, Germany) doped with the nonmesogenic azo-dye methyl-red (MR) at a $2 \%$ ratio in weigh. This ratio was identified as optimum after testing the solubility of MR in NLC and performing a probe-pump experiment by placing a NLC sample doped with MR between crossed polarizers and by measuring the transmitted intensity versus the pump power. The suitable concentration (2\%) has been chosen by combining the totally solubility of MR in NLC and the lower pump power able to induce the switch off of the transmitted intensity between crossed polarizers.

The glass and PDMS surfaces were brought into contact via $1 \mu \mathrm{m}$ glass microspheres and the $\mathrm{LC}$ was introduced by capillary action in isotropic phase $\left(65^{\circ} \mathrm{C}\right)$. The sample was brought below the isotropic-nematic transition point by means of a controlled, very slow, linear cooling down to room temperature. The employed glass substrates were both

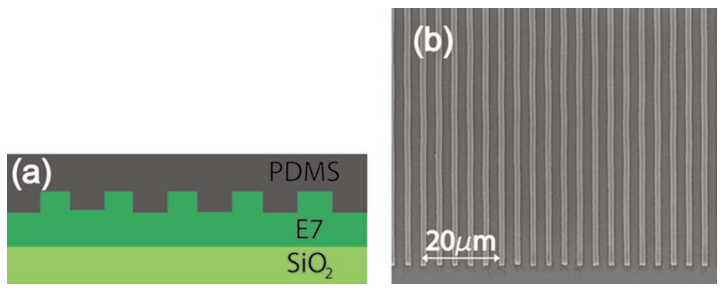

FIG. 1. (Color online) A cross-sectional schematic of the hybrid grating (a) and the corresponding SEM image of the master grating (b). 


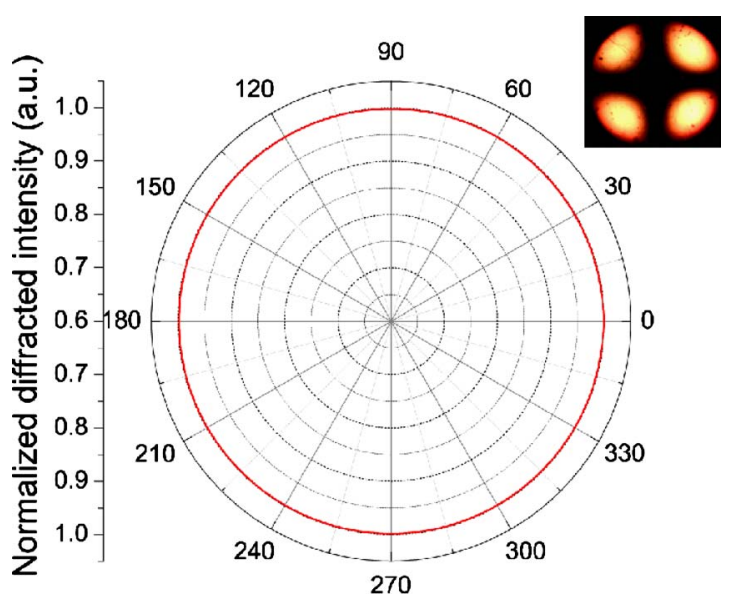

FIG. 2. (Color online) The polar diagram of the first diffracted order as a function of the polarization angle. In the inset, the conoscopic image of the PDMS-LC grating under no additional alignment layer is reported.

unmodified and coated with thin polyimide layer rubbed by means a precision rotary stage and a soft tissue in order to induce planar alignment. The PDMS grating was fabricated in two steps.

First, the master grating mold was defined on a $2 \mu \mathrm{m}$ thick SU8 film (GM-1040, Gersteltec Inc., spin speed of $1100 \mathrm{rpm}$ ) on a silicon wafer by electron beam lithography (Vistec EBPG5000, $100 \mathrm{kV}$ acceleration voltage). SU8 is a negative photoresist, but also exhibits a high sensitivity under electron beam irradiation. ${ }^{12}$ In our experiments, the clearance dose was $6 \mu \mathrm{Cb} / \mathrm{cm}^{2}$, enabling thus very short exposure duration despite the relatively large area of the grating $\left(0.25 \mathrm{~cm}^{2}\right)$. The scanning electron microscopy (SEM) picture of the master grating structure with a period of $4 \mu \mathrm{m}$ is shown in Fig. 1(b). Each line had an approximate width of $2 \mu \mathrm{m}$. The second part of the grating fabrication involved treatment of the SU8 master with trimethylchlorosilane vapor $(2 \mathrm{~min})$ and subsequently transferring the pattern of the structured SU-8 to PDMS (Dow Corning Sylgard 184) by replica molding.

The capacity of the PDMS microstructure with an unmodified cover glass to align the confined NLC was investigated by observing the dependence of the first diffracted order on the polarization state of the incident light. For this experiment, we employed a linearly polarized $\mathrm{He}-\mathrm{Ne}$ laser beam $(633 \mathrm{~nm})$, which prior to impinging on the sample at the Bragg angle, its polarization was controlled with a halfwave plate. At this wavelength we expect the transmission through the cell to be rather high, due to the low absorption cross-section of the MR. The results are shown in Fig. 2 as a function of the rotation angle of the half wave-plate, clearly indicating that the structure is polarization independent. The orientation of a birefringent material strongly influences the diffraction efficiency of the grating, which in turn depends on relative angle between the incident electric field and the average orientation of the NLC director. In our experiments, we noticed that the intensity of the first diffraction order was relatively low suggesting that the incident electric field experiences a low index contrast between the NLC and the PDMS. Such low index contrast would mostly manifest itself in the case of homeotropic alignment induced by the PDMS microstructure on the NLC component. To confirm this, we inspected the sample under a conoscopic imaging system,

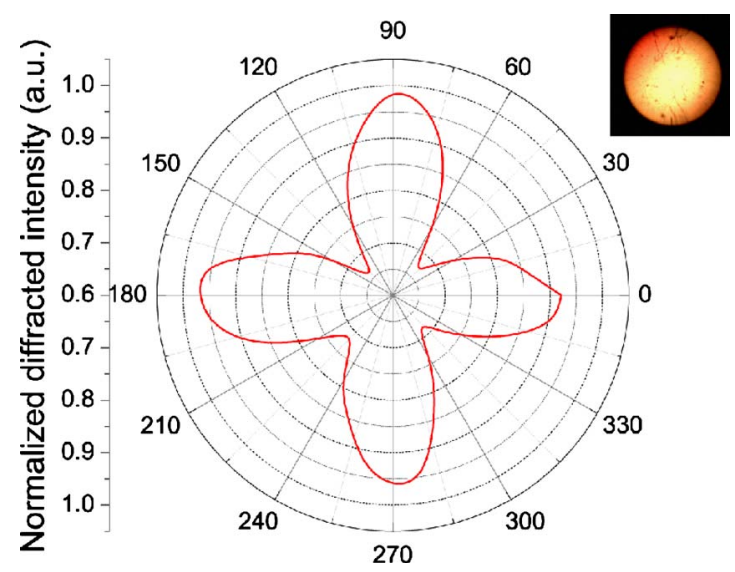

FIG. 3. (Color online) The polar diagram of the first diffracted order as a function of the polarization angle. In the inset is reported the bright spot obtained with the optical microscope in the Bertrand lens configuration.

realized by placing a conventional microscope between crossed polarizers and including a condensing and a Bertrand lens (BL) (inset of Fig. 2). At the back-focal plane of the BL we observed a dark Maltese cross. This clearly indicated that the NLC molecules are homeotropically aligned between the PDMS grating and the unmodified cover glass.

In order to induce the planar alignment of the NLC, we modified the bottom cover glass by adding a thin "polymide" layer, where a preferred planar direction was induced via rubbing. The NLC and the PDMS gratings were the same as mentioned before. In Fig. 3 the intensity of the first diffracted order is plotted as a function of the polarization angle of the incident beam. In contrast to the homeotropic alignment, the polar diagram reveals a maximum and a minimum of the diffracted intensity. This indicates that the incident light experiences a variation in the LC birefringence. We confirmed this via conoscopic imaging with an optical microscope in BL configuration (inset of Fig. 3). The image exhibits a strong variation upon rotating the sample, with the minimum intensity values being very close to zero. These observations confirm a quasiplanar NLC alignment in our hybrid grating.

To further confirm the NLC hybrid alignment, we performed an all-optical pump-probe experiment. As shown in Fig. 4, for the pump laser we used a CW diode operating at $\lambda=532 \mathrm{~nm}$. At this wavelength, MR efficiently absorbs the incident radiation and thus photoisomerizes from a cis- to trans-state. The absorption of the dye doped NLC (2\% MR in $1 \mu \mathrm{m}$ thick sample) component was investigated by means of absorption and found to be approximately $10 \%$ at the pump wavelength. For the probe beam we employed the same $\mathrm{He}-\mathrm{Ne}$ laser as previously, operating at a wavelength of $\lambda=633 \mathrm{~nm}$. The probe beam impinges on the sample at an angle of $2^{\circ}$, which corresponds to the Bragg angle. The probe

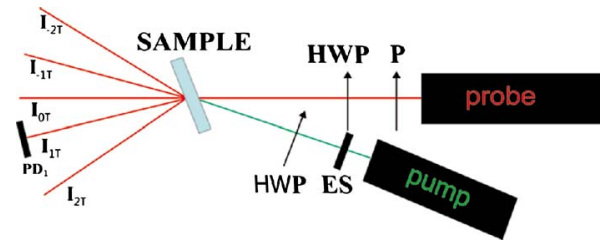

FIG. 4. (Color online) The experimental setup for the observation of alloptical switching. $\mathrm{PD}_{1}$ : photodetector; HWP: half-wave plate; P: polarizer; ES: electronic shutter. 


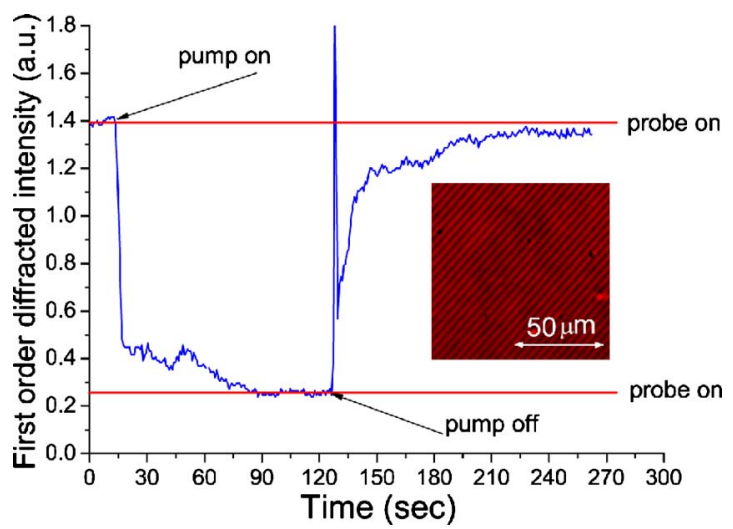

FIG. 5. (Color online) Changing in the first-order diffracted intensity by switching on and off the pump beam. In the inset is reported an optical microscope view of the structure.

was additionally p-polarized in order to experience the highest index contrast, which in our experiments we estimate to be 0.15 . The excitation area was $2 \times 1 \mathrm{~mm}^{2}$, corresponding to the patterned area of the grating. The powers of the pump and probe beams were $20 \mathrm{~mW}$ and $4 \mathrm{~mW}$, respectively. The grating operates in the Raman-Nath regime and thus exhibits a multi-order diffraction pattern. To perform all-optical switching, we monitored the intensity variation in the first diffracted order.

When the hybrid grating is excited by the pump beam, the NLC molecules become disordered, due to the trans-cis photoisomerization process, and the probe experiences the average refractive index. This index is very close to the one of PDMS and thus causes the diffracted intensity to significantly decrease. This state corresponds to the first $15 \mathrm{~s}$ in Fig. 5. When the pump beam is switched off (130 s in Fig. 5 ), cis-trans photoisomerization of the azo-dye takes place, which in turn induces orientation of the NLC director and the strong diffraction is re-established. The diffraction efficiency (defined as the ratio of the diffracted intensity over the impinging intensity) was measured to be approximately $20 \%$. The peak in the first order diffracted intensity observed at the time of turning the pump beam off (Fig. 5) reached to $35 \%$. The reason of this big jump is due to the competition between the high ordered state induced by cis-trans back isomerization process (fast response) and the boundary conditions imposed by the surface and grating walls (long term response).
However, due to the low pump power density used in our experiments $\left(0.6 \mathrm{~W} / \mathrm{cm}^{2}\right)$, we consider negligible the local heating (isothermal phase transition) induced by the pump beam on the dye doped NLC component as reported in. ${ }^{13-15}$ We attribute the variation in the diffracted intensity (Fig. 5) to the local change of the order parameter induced by the trans-cis photoisomerization process of the MR.

In conclusion, we have reported the observation of alloptical control device realized by cast-molding lithography in PDMS. The value of the diffraction efficiency is not the highest reported in the literature of composite diffraction gratings ${ }^{16}$ and current ongoing investigations aim at enhancing this efficiency by increasing the NLC alignment (fullplanar condition) and employing shorter grating periodicity. In addition, to improve the response time, mesogenic azodye materials ${ }^{17}$ will be investigated by using as pump a suitable pulsed laser.

${ }^{1}$ A. Urbas, J. Klosterman, V. Tondiglia, L. Natarajan, R. Sutherland, O. Tsutsumi, T. Ikeda, and T. Bunning, Adv. Mater. 16, 1453 (2004).

${ }^{2}$ L. De Sio, A. Veltri, C. Umeton, S. Serak, and N. Tabirian, Appl. Phys. Lett. 93, 181115 (2008).

${ }^{3}$ Y. J. Wang and Z. Guo, Opt. Eng. 34, 1482 (1995).

${ }^{4} \mathrm{H}$. Knoll, in CRC Handbook of Organic Photochemistry and Photobiology, 2nd ed., edited by F. Lenci and W. Horspool, (CRC, Boca Raton, 2004).

${ }^{5}$ U. A. Hrozhyk, S. V. Serak, N. V. Tabiryan, L. Hoke, D. M. Steeves, B. Kimball, and G. Kedziora, Mol. Cryst. Liq. Cryst. 489, 257 (2008).

${ }^{6}$ N. A. Mortensen, S. Xiao and J. Pedersen, Microfluid. Nanofluid. 4, 4982 (2008).

${ }^{7}$ D. Psaltis, S. R. Quake, and C. H. Yang, Nature (London) 442, 381 (2006).

${ }^{8}$ C. Monat, P. Domachuk, and B. J. Eggleton, Nat. Photonics 1, 106 (2007). ${ }^{9}$ M. Humar, M. Ravnik, S. Pajk, and I. Musevic, Nat. Photonics 3, 595 (2009)

${ }^{10}$ J. Y. Woo, E. H. Kim, and B. K. Kim, ChemPhysChem 9, 141 (2008).

${ }^{11}$ S. J. Woltman, G. D. Jay, and G. P. Crawford, Nature Mater. 6, 929 (2007).

${ }^{12}$ B. Bilenberg, S. Jacobsen, M. S. Schmidt, L. H. D. Skjolding, P. Shi, P. Boggild, J. O. Tegenfeldt, and A. Kristensen, Microelectron. Eng. 83, 1609 (2006).

${ }^{13}$ C. Umeton, A. Sgrò, and F. Simoni, J. Opt. Soc. Am. B 4, 1938 (1987).

${ }^{14} \mathrm{P}$. G. de Gennes and J. Prost, The Physics of Liquid Crystals, 2nd ed. (Oxford University Press, New York, 1993).

${ }^{15}$ P. Jagemalm, D. S. Hermann, L. Komitov, and F. Simoni, Liq. Cryst. 24 335 (1998).

${ }^{16}$ L. De Sio, S. Serak, N. Tabiryan, S. Ferjani, A. Veltri, and C. Umeton, "Composite holographic gratings containing light responsive liquid crystals for visible bichromatic switching," Adv. Mater. (to be published).

${ }^{17}$ U. Hrozhyk, S. Nersisyan, S. Serak, N. Tabiryan, L. Hoke, D. Steeves, and B. Kimball, Opt. Lett. 34, 2554 (2009). 